\title{
Living System Adapts Harmonics of Peristaltic Wave for Cost-Efficient Optimization of Pumping Performance
}

\author{
Felix K. Bäuerle, ${ }^{1}$ Stefan Karpitschka, ${ }^{1}$ and Karen Alim $\circledast^{1,2, *}$ \\ ${ }^{1}$ Max Planck Institute for Dynamics and Self-Organization, 37077 Göttingen, Germany \\ ${ }^{2}$ Physik Department, Technische Universität München, 85748 Garching b. München, Germany
}

(Received 19 December 2018; accepted 3 January 2020; published 5 March 2020)

\begin{abstract}
Wavelike patterns driving transport are ubiquitous in life. Peristaltic pumps are a paradigm of efficient mass transport by contraction driven flows-often limited by energetic constraints. We show that a costefficient increase in pumping performance can be achieved by modulating the phase difference between harmonics to increase occlusion. In experiments we find a phase difference shift in the living peristalsis model $P$. polycephalum as dynamic response to forced mass transport. Our findings provide a novel metric for wavelike patterns and demonstrate the crucial role of nonlinearities in life.
\end{abstract}

DOI: 10.1103/PhysRevLett.124.098102

Wavelike patterns are ubiquitous in life. They range from everyday phenomena such as gut peristalsis [1], undulatory locomotion of wormlike organisms [2], or the cardiac cycle [3], to microscopic waves, such as ciliary and flagellar beating [4], gene oscillation [5], or reaction-diffusion patterns [6]. The patterns' functions vary, but it is noteworthy that they often relate directly to transport or locomotion. Each system oscillates in distinct characteristics, for example, body shape [7] or molecule concentration [8], yet all systems are governed by the limited set of a wave's parameters - wavelength, amplitude, and frequency. Moreover parameter choice is limited by physical or biological constraints. Which strategies does a living system use to fulfill the wave's function given its constraints?

Environmental changes challenge living systems to adapt, forcing alteration of wave dynamics given the limited set of wave parameters while additionally staying within its constraints. As an example, the nematode C.elegans changes its locomotion depending on the viscoelasticity of its environment by modulating undulation wavelength, amplitude, and frequency of its wave-shaped body [9]. Yet, this adaptation goes hand in hand with changes in the energy cost of the wave, often the most limiting constraint of life [10,11].

While a sinusoidal wave shape offers few parameters to adjust, some living systems instead use a superposition of waves. Examples are the peristaltic contractions of the human gut [12] or the flagellar beating of human spermatozoa [13]. The superposition of multiple waves allows a modulation of the total wave shape, thereby increasing the

Published by the American Physical Society under the terms of the Creative Commons Attribution 4.0 International license. Further distribution of this work must maintain attribution to the author(s) and the published article's title, journal citation, and DOI. Funded by the Max Planck Society. parameter space available when responding to environmental changes. In particular, for a set of harmonic waves, we here introduce the phase difference between them as a new parameter. How much control over the waves' total performance resides in the phase difference between superposed harmonic waves given limited wave energy cost?

The paradigm for wave driven mass transport and locomotion is peristaltic pumping [14,15]. Here, mass transport is driven by radial contractions traveling along a tube which cause net transport of the enclosed fluid, see Fig. 1. The contractions can be considered as periodic train waves with the characteristic wave parameters defining the flow rate. To this point it has been unclear how peristaltic pumping performance can increase in a cost efficient manner, since changing the wave's symmetry has been
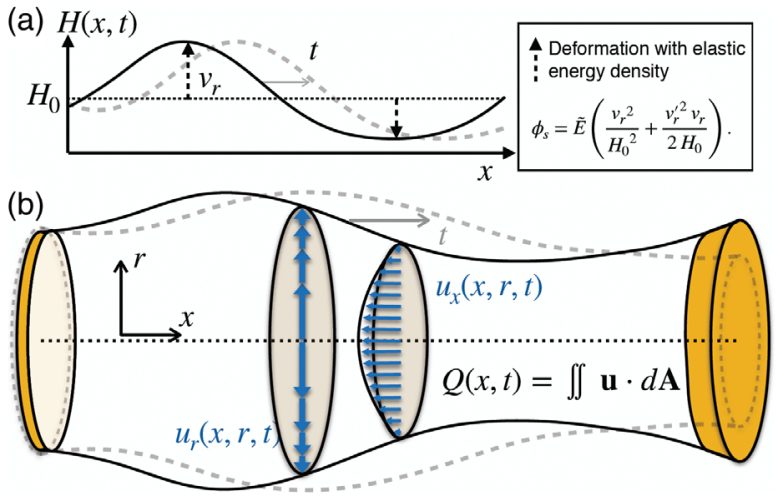

FIG. 1. Scheme of a tube as a peristaltic pump and its elastic energy. (a) Deformation of the tube boundary $H(x, t)=H_{0}+v_{r}$ from the rest radius $H_{0}$ increases the elastic energy proportionally to the stretching energy density $\phi_{s}$ with a prefactor $\tilde{E}$. (b) Scheme of a tube acting as a peristaltic pump. The deformation of the tube boundary $H(x, t)$ over time drives radial, $u_{r}$, and axial, $u_{x}$, flow velocities and thus flow rate $Q(x, t)$ in the tube. 
shown to have a negligible effect on the pumping performance [16]. To resolve this puzzle we turn to living systems forced to transport mass in response to environmental cues.

The network-forming slime mold Physarum polycephalum has gained broad attention as a living system using peristalsis [17-19]. Its interconnected tubes contract rhythmically, driving fluid flow throughout the network [19]. The tube walls are made of acto-myosin and behave as an active viscoelastic material pumping the cytoplasmic fluid back and forth in a shuttle flow [20,21]. In response to environmental changes, the slime mold rapidly migrates toward attractive stimuli or away from repellents, efficiently turning the periodic shuttle flow into a net mass transport [22-25]. For $P$. polycephalum the dominant contraction frequency and its second harmonic make up the bulk of its oscillation modes which render it an ideal candidate to investigate the control of wave parameters on pumping performance in a living system [26].

In this Letter, we investigate how a living system can control its waves' performance in a cost-efficient manner. Specifically, we study the performance of a peristaltic pump by evaluating the elastic deformation energy of its contraction wave. The wave train consists of a dominant wave and its second harmonic introducing the phase difference between them as an additional adjustable parameter. We analytically show that the flow rate can be controlled by adjusting the phase difference while optimizing the required elastic energy for deformation. At the optimal phase difference the resulting wave shape occludes the tube most tightly, which optimizes pumping performance by over $25 \%$ under physiological conditions. Investigating the peristaltic waves in $P$. polycephalum, we initially find that unforced specimens favor minimal occlusion. Only when forced to transport mass by a phototactic stimulus, specimens adjust their phase differences toward maximal occlusion optimal for pumping performance. Here, the phase difference serves as a subtle but powerful parameter, in line with our theoretical predictions. To elucidate how the living system self organizes its pumping performance we discuss nonlinear tube wall viscoelasticity or active processes as a putative control for the phase difference adaption.

A peristaltic pump drives the directed transport of fluid through a circular tube by a periodic wave train of contractions $[14,15]$. We consider a single tube of shape $H(x, t)$ and length $L$ filled with an incompressible Newtonian fluid extending along longitudinal $x$ and radial $r$ coordinates, see Fig. 1. In the limit of a long slender tube $L \gg H(x, t)$ the lubrication approximation applies [27], simplifying the equations governing the axial and radial flow velocity, $u_{x}(x, r, t)$ and $u_{r}(x, r, t)$, respectively, to

$$
\frac{\partial p}{\partial x}=\frac{\mu}{r} \frac{\partial}{\partial r}\left(r \frac{\partial u_{x}}{\partial r}\right), \quad \frac{1}{r} \frac{\partial\left(r u_{r}\right)}{\partial r}+\frac{\partial u_{x}}{\partial r}=0
$$
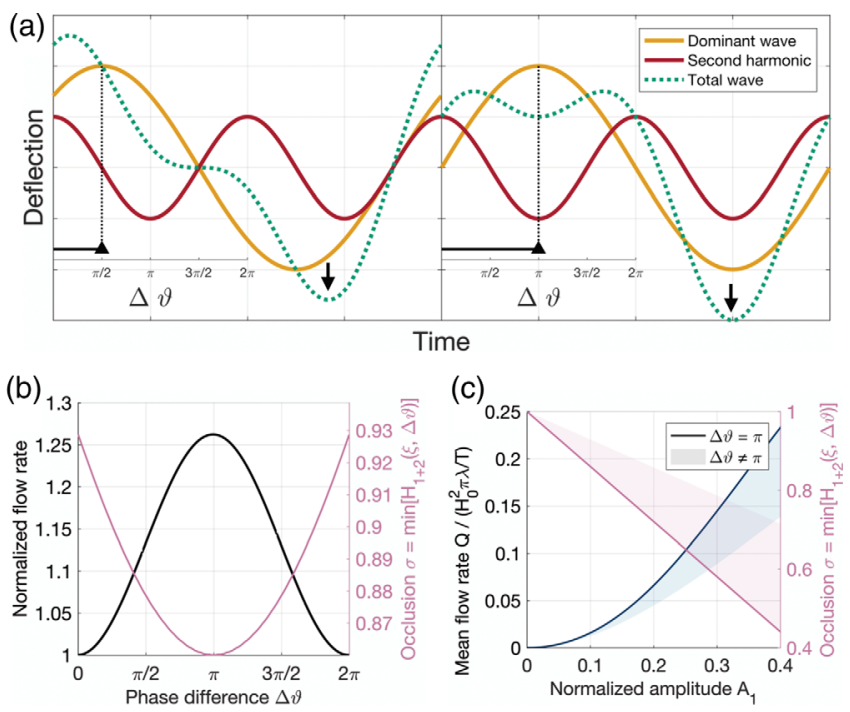

FIG. 2. Phase difference $\Delta \vartheta$ between a dominant wave and its second harmonic controls flow rate by adjusting occlusion. (a) Scheme of dominant wave (yellow) and second harmonic (red) and their superposition (green dashed). Occlusion is maximal for $\Delta \vartheta=\pi$, where the minima of the dominant wave and second harmonic align (right panel, compare arrows). (b) Flow rate and occlusion of $H_{1+2}$ wave, Eq. (3), vs the phase difference shows maximal flow rate at $\Delta \vartheta=\pi$; amplitudes represent physiological values for $P$. polycephalum $A_{1}=0.1 H_{0}$ and $A_{2}=0.04 H_{0}$. (c) Dependence of flow rate (blue) and occlusion (pink) of $H_{1+2}$ wave on amplitude $A_{1}$ and phase difference (shaded area). Second harmonic amplitude is fixed at $A_{2}=0.4 A_{1}$ as above. Solid line highlights optimal condition at $\Delta \vartheta=\pi$.

where $p(x, t)$ denotes the pressure and $\mu$ the fluid's viscosity. Specifying a no-slip boundary condition at the wall then fully defines the flow as a function of pressure gradient $\partial p / \partial x$ and tube wall shape $H(x, t)$. The pumping performance solely due to the periodic wave train, i.e., without an applied pressure gradient, is then specified by the time-averaged volume flow rate $\bar{Q}_{0}$ over the wave period $T$ [15],

$$
\bar{Q}_{0}=\frac{2 \pi}{T} \int_{0}^{T} \frac{\int_{0}^{L} H^{-4}\left(x_{2}, t\right) \int_{0}^{x_{2}} \frac{\partial H^{2}\left(x_{1}, t\right)}{\partial t} d x_{1} d x_{2}}{\int_{0}^{L} H^{-4}(x, t) d x} d t .
$$

To investigate how higher harmonics affect the pumping performance we assume the wall shape to be a superposition of two sinusoidal waves: a dominant wave and its second harmonic,

$$
H_{1+2}(\xi, \Delta \vartheta)=H_{0}+A_{1} \cos (2 \pi \xi)+A_{2} \cos (4 \pi \xi+\Delta \vartheta),
$$

where $\xi(x, t)=x / \lambda-t / T$, with $\lambda$ the wavelength of the dominant wave, and $\Delta \vartheta$ the phase difference of the second harmonic with respect to the dominant wave, see Fig. 2(a). Note, that the wavelength $\lambda$ and tube length $L$ can differ, 
but its effect is negligible on the pumping performance for $L \geq \lambda[15] . H_{0}$ is the radius baseline around which the tube oscillates and $A_{1}$ and $A_{2}$ are the amplitudes of the dominant wave and its second harmonic, respectively. Furthermore, the tube's thin wall gets deformed from its baseline $H_{0}$ and stores a total elastic energy, see the Supplemental Material [28] for derivation,

$$
\bar{\Phi}[H(x, t)]=\frac{E h H_{0} \pi}{\left(1-\nu^{2}\right)} \int_{0}^{L}\left(\frac{v_{r}^{2}}{H_{0}^{2}}+\nu \frac{v_{r}^{\prime 2}}{H_{0}} v_{r}\right) d x,
$$

with $v_{r}(x, t)=H(x, t)-H_{0}$ the radial deformation, $\nu$ Poisson's ratio, $h$ the tube wall thickness, and $E$ the Young's modulus. Note that the contraction wavelength $\lambda$ is much larger than the tube's radius in $P$. polycephalum [19], which renders bending elastic energy along the axis negligible in comparison to radial deformation considered here; see Supplemental Material [28] for additional information. Therefore, the elastic energy per fundamental wave for the given tube shape $H_{1+2}$ and a Poisson's ratio of $\frac{1}{2}$ is

$$
\bar{\Phi}\left(H_{1+2}\right)=\frac{2 \pi}{3} E h\left(\frac{A_{1}^{2}}{H_{0}}+\frac{A_{2}^{2}}{H_{0}}+\frac{3 \pi^{2} A_{2} A_{1}^{2}}{\lambda^{2}} \cos (\Delta \vartheta)\right) .
$$

Following Eq. (5) the waves' amplitudes $A_{1}$ and $A_{2}$ dominate the elastic energy. The phase difference $\Delta \vartheta$ only contributes to higher order, scaling inversely with $\lambda^{2}$. For constant amplitudes the energy is minimized for $\Delta \vartheta=\pi$ and maximized for $\Delta \vartheta=(0,2 \pi)$, regardless of the wavelength of the contraction wave. Despite the negligible contribution to the elastic energy, varying the phase difference $\Delta \vartheta$ changes the waveform, see Fig. 2(a), and most notably dramatically alters the resulting flow rate following Eq. (2), see Fig. 2(b). Flow rate is maximal for $\Delta \vartheta=\pi$, and minimal for $\Delta \vartheta=(0,2 \pi)$. Considering physiological amplitudes of $A_{1}=0.1 H_{0}$ and $A_{2}=0.04 H_{0}$, see Supplemental Material [28], the phase difference shift can increase the flow rate by over $25 \%$. Hence, pumping performance of a peristaltic pump can be optimized with a cost-efficient shift towards a phase difference of $\Delta \vartheta=\pi$, thereby even minimizing elastic deformation energy. Note, that this result for a single tube can be extended to networks formed by $P$. polycephalum as peristaltic waves span the entire network with no dispersion at the vertices, particularly in the networks selected for homogeneous architecture presented in the following, see Supplemental Material [28].

We identify the tighter occlusion of the tube, i.e., the alignment of the minima of the first and the second harmonic for $\Delta \vartheta=\pi$, as the reason for the increased flow rate, see Figs. 2(b) and 2(c). To gain intuition why a tighter occlusion increases the flow rate, one can interpret the flow rate in Eq. (2) as a weighted average with the weight given by $H^{-4}(x, t)$. Even though the weighted term is periodic in space and time, the total weight is increased

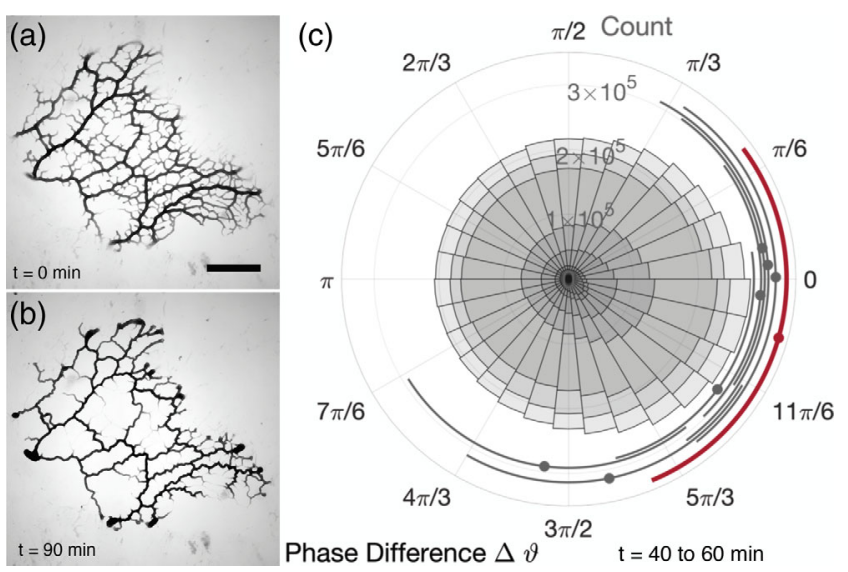

FIG. 3. Unforced $P$. polycephalum has a phase difference distribution centered around $\Delta \vartheta=0$ corresponding to minimal occlusion. (a),(b) Brightfield images of the unforced $P$. polycephalum network 90 min apart. Bar $=2 \mathrm{~mm}$. (c) Stacked polar histogram of the phase difference $(n=7)$ between 40 to 60 min after the start of data acquisition. Dots indicate mean and lines $33 \%$ intervals. The mean and interval for the total distribution is shown in red.

for small $H(x, t)$, i.e., tighter occlusion, which increases the average flow rate maximized at $\Delta \vartheta=\pi$.

Having derived in theory how big the effect of a shift in phase difference between dominant wave and second harmonic can be, we turn to quantify this defining wave parameter in a living system. Our model system here is $P$. polycephalum, an organism gaining attention as a living network-shaped peristaltic pump. In fact, all individual tubes of the network undergo peristaltic contraction.

P. polycephalum (Carolina Biological Supply Company) networks were prepared with an oat flake or microplasmodia culture on $1.5 \%$ agar plates [29,39]. Networks were recultured every three days for oat flake culture. After an overnight culture, the networks were selected for homogeneous tube diameter and segment length and then cut to a size of roughly $30 \mathrm{~mm}^{2}$ with an inoculation loop $90 \mathrm{~min}$ before data acquisition with 3 to $6 \mathrm{sec}$ per frame to mitigate any reaction from cutting [29]. Peristaltic contraction dynamics throughout the network are quantified from brightfield images with a custom written Matlab program [29]. To quantify the phase difference $\Delta \vartheta$ between the second harmonic and the dominant wave in the networkwide contractions we first map out each tube's contractions. The contractions' traces over time are high-pass filtered and band-pass filtered for the dominant and the second harmonic, respectively. The phases of the two waves are isolated with a stepwise fitting of the corresponding waveform to the filtered data; see Supplemental Material [28] for an in-depth description.

Unforced networks starve over time and as a response move mass from central, small tubes to the periphery without a preferred direction [40]. Figure 3 shows the network-wide distribution of phase differences $\Delta \vartheta$. Contrary to our 

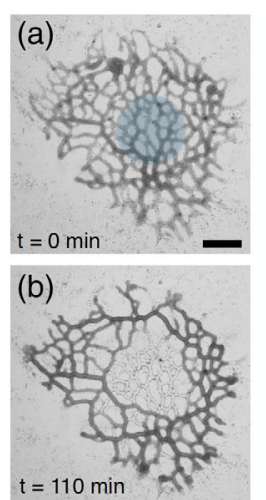

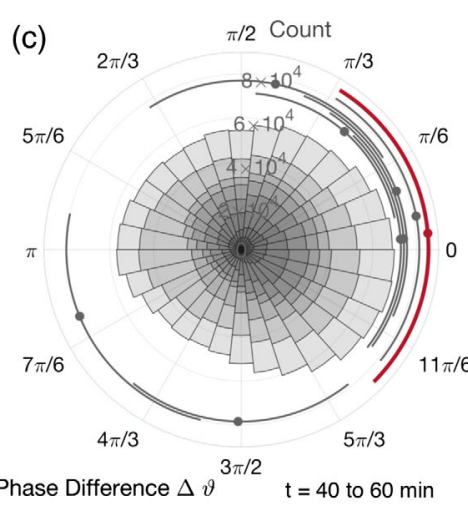

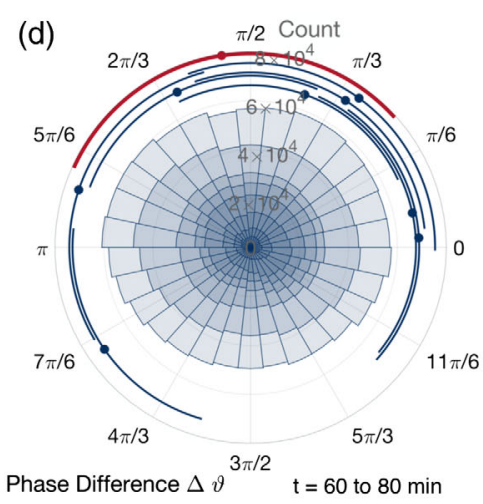

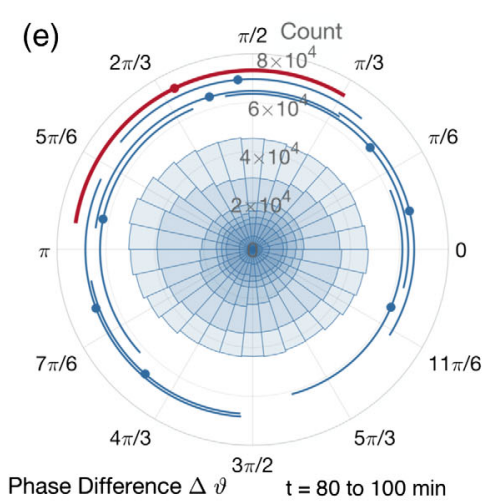

FIG. 4. P. polycephalum adapts its phase difference $\Delta \vartheta$ when forced to increase mass transport. (a),(b) $P$. polycephalum networks with the illuminated region (blue) before (a) and after (b) the onset of a blue light stimulus (at $t=60 \mathrm{~min}$ ). Bar $=1 \mathrm{~mm}$. (c),(d), (e) Stacked polar histogram distributions of phase difference $\Delta \vartheta$ for light enforced experiments in three consecutive time windows $(n=8 ;(\mathrm{c}) \hat{=} 40$ to $60,(\mathrm{~d}) \hat{=} 60$ to $80,(\mathrm{e}) \hat{=} 80$ to $100 \mathrm{~min}$ ). Colored dots indicate mean and lines $33 \%$ intervals. The mean and interval for the total distribution is shown in red.

expectations of life being optimized for flow rate, unforced $P$. polycephalum networks show a phase difference distribution peaked around $\Delta \vartheta=0$, the point of minimal occlusion $(n=7)$.

Being inspired by Purcell's observation [41] that living organisms may only optimize their most pressing problem we next force $P$. polycephalum to transport mass by providing a phototactic stimulus. $P$. polycephalum's phototactic response is wavelength dependent, with the strongest avoidance reaction to blue light at around $450 \mathrm{~nm}$ [42]. We applied blue light with a halogen lamp (Illuminator HXP 200C) and a filter set (Zeiss $38 \mathrm{HE}$, excitation BP 470/40) continuously from light onset, designed to trigger mass transport away from the networks' center. Illuminated regions were fully surrounded by network and are chosen to have a size of roughly $30 \%$ to $50 \%$ of the total network size at the start of recording. Image recording started $60 \mathrm{~min}$ before light exposure allowing us to track phase shift evolution over time before complete relocation from the illuminated region, see Figs. 4(a) and 4(b). We bin the distribution of phase differences $\Delta \vartheta$ into time windows of 20 min, once right before the onset of illumination and two sets after onset of illumination, see Figs. 4(c)-4(e). Confirming our previous observations of unforced specimens the phase difference distributions before the onset of illumination are peaked around $\Delta \vartheta=0$. Yet, forcing the specimen with light to increase mass transport we now observe that under blue light illumination the phase difference successively shifts toward $\Delta \vartheta=\pi$, the optimal phase difference for peristaltic pumping; see also Supplemental Material [28]. Why is the second harmonic aligned inefficiently around $\Delta \vartheta=0$ when unforced? And does the living system actively adjust the second harmonic of its peristaltic wave for higher pumping performance?

Our experimental approach allows us to quantify the tube deformation, but not if the deformations' second harmonic is arising actively or passively. Therefore, from data we cannot infer if the surprising adjustment of phase difference following forcing is an active adaptation of contractions of the living system. It is, however, instructive to compare our observations to the wave shape forming in a viscoelastic tube filled with a viscous fluid driven solely by a dominant wave $F_{P} \sim \cos (2 \pi \xi)$. The tube responds with a second harmonic in deformation arising passively: Following the approach of Takagi and Balmforth [37] and assuming a weakly viscoelastic restoring force $F_{E}=\left\{E h /\left[\left(1-\nu^{2}\right) H_{0}^{2}\right]\right\} v_{r}+\eta\left(\partial v_{r} / \partial t\right)$, with dampening coefficient $\eta$, we expand systematically for small $v_{r}$ as in Ref. [38] to derive dominant and second harmonic of the tube deformation, see Supplemental Material [28]. Their phase difference is $\Delta \vartheta=(\pi / 2)-$ $\arctan \left\{\left[4\left(1-\nu^{2}\right) / E h T\right]\left[\left(\mu \lambda^{2} / \pi H_{0}\right)+\pi \eta H_{0}^{2}\right]\right\}$ and is constrained between $\Delta \vartheta \in[0,(\pi / 2)]$. For physiological parameters (see Supplemental Material [28]), $\Delta \vartheta \sim 0.1$ close to the observed minimal occlusion in unforced networks. Thus, the nonoptimal $\Delta \vartheta \sim 0$ in unforced data is in agreement with a fluid-filled viscoelastic tube with fundamental forcing only. Furthermore, a phase difference outside the range $[0,(\pi / 2)]$ can result only from nonlinear material behavior or active forces. Strain hardening would drive the phase difference toward $\pi$, in line with our observations for forced specimen, see Supplemental Material [28]. Even though geometric nonlinear effects remain small, an adaptive nonlinear material property may be a convoluted, yet surprisingly efficient way to adapt the phase difference and therewith pumping efficiency. Alternatively the slime mold may actively control its phase difference, i.e., by utilizing two separate oscillators with an adaptive phase relation. The high, and surprisingly stable, amplitude ratio $A_{2} / A_{1}$ is indicative for the latter, with the passively arising overtone acting as a reference point when unforced.

Investigating how living systems can control their wave's performance in a cost efficient manner we here identified 
the phase difference between wave harmonics as a subtle but powerful parameter. We find that the performance of peristaltic pumping can be enhanced by over $25 \%$ while lowering the elastic energy cost by optimizing the phase difference between the dominant wave and its second harmonic of the contraction wave. Testing for this mechanism in the slime mold $P$. polycephalum, we observe a shift in phase difference toward optimal pumping performance when forced to transport mass in response to environmental changes. We identify a passive viscoelastic response as a putative source for the overtone and find that nonlinear material behavior can adapt the phase difference efficiently.

Our findings spotlight the important role of higher harmonics in wave phenomena. Higher harmonics intrinsically arise in numerous systems like coupled oscillators [43] or active viscoelastic materials [33,37]. Living systems, as exemplified here, use the phase difference between harmonics as a key cost efficient parameter to adjust wave performance. Specifically, control may reside in the inherent nonlinear material properties that may change upon environmental stimuli [44]. It is inspiring to speculate that life's biochemical machinery can quickly adjust its viscoelastic properties of an actin cortex, e.g., by controlling the access to globular actin via secondary proteins [45]. A control that could prove powerful to synthetic biology. In conclusion, our findings provide a novel metric for wave phenomena in general, namely, the phase difference between wave harmonics. We demonstrate the crucial role and control residing in nonlinearities of living systems. Our life-inspired findings on flow control in peristaltic pumping may find direct application in the design of smart materials and soft robotics.

We thank Holger Nobach for stimulating discussions regarding time-series analysis and Leonie Bastin for providing data of the unforced specimen. This work was supported by the Max Planck Society. F. B. acknowledges support by the International Max Planck Research School for Physics of Biological and Complex Systems.

\section{*k.alim@tum.de}

[1] J. D. Huizinga and W. J. E. P. Lammers, Am. J. Physiol. Gastrointest. Liver Physiol. 296, G1 (2009).

[2] N. Cohen and J. H. Boyle, Contemp. Phys. 51, 103 (2010).

[3] J. M. Davidenko, A. V. Pertsov, R. Salomonsz, W. Baxter, and J. Jalife, Nature (London) 355, 349 (1992).

[4] S. Camalet, F. Jülicher, and J. Prost, Phys. Rev. Lett. 82, 1590 (1999).

[5] D. Bell-Pedersen, V. M. Cassone, D. J. Earnest, S. S. Golden, P. E. Hardin, T. L. Thomas, and M. J. Zoran, Nat. Rev. Genet. 6, 544 (2005).

[6] S. Kondo and T. Miura, Science 329, 1616 (2010).

[7] R. M. Alexander, Physiol. Rev. 69, 1199 (1989).

[8] M. W. Berchtold, H. Brinkmeier, and M. Müntener, Physiol. Rev. 80, 1215 (2000).
[9] S. Berri, J. H. Boyle, M. Tassieri, I. A. Hope, and N. Cohen, HFSP J. 3, 186 (2009).

[10] N. Osterman and A. Vilfan, Proc. Natl. Acad. Sci. U.S.A. 108, 15727 (2011).

[11] Y. Katsu-Kimura, F. Nakaya, S. A. Baba, and Y. Mogami, J. Exp. Biol. 212, 1819 (2009).

[12] J. D. Huizinga et al., Nat. Commun. 5, 3326 (2014).

[13] G. Saggiorato, L. Alvarez, J. F. Jikeli, U. B. Kaupp, G. Gompper, and J. Elgeti, Nat. Commun. 8, 1415 (2017).

[14] A. H. Shapiro, M. Y. Jaffrin, and S. L. Weinberg, J. Fluid Mech. 37, 799 (1969).

[15] M. Li and J. G. Brasseur, J. Fluid Mech. 248, 129 (1993).

[16] O. Mahrenholtz, M. Mank, and R. Zimmermann, Biorheology 15, 501 (1978).

[17] C. Oettmeier, K. Brix, and H.-G. Döbereiner, J. Phys. D 50, 413001 (2017).

[18] M. Iima and T. Nakagaki, Math. Med. Biol. 29, 263 (2012).

[19] K. Alim, G. Amselem, F. Peaudecerf, M. P. Brenner, and A. Pringle, Proc. Natl. Acad. Sci. U.S.A. 110, 13306 (2013).

[20] W. Stockem and K. Brix, in International Review of Cytology, Vol. 149 (Elsevier, New York, 1994), pp. 145-215.

[21] A. Fessel, C. Oettmeier, K. Wechsler, and H.-G. Döbereiner, J. Phys. D 51, 024005 (2018).

[22] S. Kuroda, S. Takagi, T. Nakagaki, and T. Ueda, J. Exp. Biol. 218, 3729 (2015).

[23] O. L. Lewis, S. Zhang, R. D. Guy, and J. C. del Alamo, J. R. Soc. Interface 12, 20141359 (2015).

[24] Y. Miyake, H. Tada, M. Yano, and H. Shimizu, Cell Struct. Funct. 19, 363 (1994).

[25] Z. Hejnowicz and K. E. Wohlfarth-Bottermann, Planta 150, 144 (1980).

[26] S. G. Proskurin and T. I. Avsievich, Biophysics 59, 928 (2014).

[27] A. Oron, S. H. Davis, and S. G. Bankoff, Rev. Mod. Phys. 69, 931 (1997).

[28] See Supplemental Material at http://link.aps.org/ supplemental/10.1103/PhysRevLett.124.098102 for an indepth description of the phase difference determination including Refs. [19,29], for the time development of individual experiments, for a description of the used physiological parameters including Refs. [19,30-34], for quantification of the spatial wave patterns in forced experiments including Ref. [19], for the derivation of the elastic deformation model including Refs. [35,36], and for the derivation of a perturbative approach on peristaltically contracting tubes including Refs. [37,38].

[29] F. K. Bäuerle, M. Kramar, and K. Alim, J. Phys. D 50, 434005 (2017).

[30] R. Swaminathan, C. Hoang, and A. Verkman, Biophys. J. 72, 1900 (1997).

[31] W. Naib-Majani, V. A. Teplov, and Z. Baranowski, in Cell Dynamics, Protoplasma Vol. 1, edited by M. Tazawa (Springer, New York, 1988), pp. 57-63.

[32] G. Salbreux, G. Charras, and E. Paluch, Trends Cell Biol. 22, 536 (2012).

[33] S. Alonso, M. Radszuweit, H. Engel, and M. Bär, J. Phys. D 50, 434004 (2017).

[34] I. Block and K. Wohlfarthbottermann, Cell Biol. Int. Rep. 5, 73 (1981). 
[35] B. Audoly and Y. Pomeau, Elasticity and Geometry, From Hair Curls to the Non-linear Response of Shells (Oxford University Press, Oxford, 2010).

[36] J. L. J. Sanders, Q. Appl. Math. 21, 21 (1963).

[37] D. Takagi and N. J. Balmforth, J. Fluid Mech. 672, 196 (2011).

[38] A. Pandey, S. Karpitschka, C. Venner, and J. Snoeijer, J. Fluid Mech. 799, 433 (2016).

[39] C. Westendorf, C. J. Gruber, K. Schnitzer, S. Kraker, and M. Grube, J. Phys. D 51, 344001 (2018).
[40] S. Marbach, K. Alim, N. Andrew, A. Pringle, and M. P. Brenner, Phys. Rev. Lett. 117, 178103 (2016).

[41] E. Purcell, Am. J. Phys. 45, 3 (1977).

[42] Y. Mori, K. Matsumoto, T. Ueda, and Y. Kobatake, Protoplasma 135, 31 (1986).

[43] T. Saigusa, A. Tero, T. Nakagaki, and Y. Kuramoto, Phys. Rev. Lett. 100, 018101 (2008).

[44] J.-D. Julien and K. Alim, Proc. Natl. Acad. Sci. U.S.A. 115, 10612 (2018).

[45] T. P. Stossel, J. Cell Biol. 99, 15s (1984). 